ARTICLE

DOI: $10.1038 / s 41467-017-01813-9$

\title{
Biosynthesis of helvolic acid and identification of an unusual C-4-demethylation process distinct from sterol biosynthesis
}

\author{
Jian-Ming Lv', Dan Hu (1) 1, Hao Gao (1) 1, Tetsuo Kushiro², Takayoshi Awakawa², Guo-Dong Chen', \\ Chuan-Xi Wang (10) ${ }^{1}$, Ikuro Abe (i) ${ }^{2} \&$ Xin-Sheng Yao ${ }^{1}$
}

Fusidane-type antibiotics represented by helvolic acid, fusidic acid and cephalosporin $\mathrm{P}_{1}$ are a class of bacteriostatic agents, which have drawn renewed attention because they have no cross-resistance to commonly used antibiotics. However, their biosynthesis is poorly understood. Here, we perform a stepwise introduction of the nine genes from the proposed gene cluster for helvolic acid into Aspergillus oryzae NSAR1, which enables us to isolate helvolic acid $\left(\sim 20 \mathrm{mg} \mathrm{L}^{-1}\right)$ and its 21 derivatives. Anti-Staphylococcus aureus assay reveals that the antibacterial activity of three intermediates is even stronger than that of helvolic acid. Notably, we observe an unusual C-4 demethylation process mediated by a promiscuous short-chain dehydrogenase/reductase (HelC) and a cytochrome P450 enzyme (HelB1), which is distinct from the common sterol biosynthesis. These studies have set the stage for using biosynthetic approaches to expand chemical diversity of fusidane-type antibiotics.

\footnotetext{
${ }^{1}$ Institute of Traditional Chinese Medicine and Natural Products, College of Pharmacy/Guangdong Province Key Laboratory of Pharmacodynamic Constituents of TCM and New Drugs Research, Jinan University, Guangzhou 510632, People's Republic of China. ${ }^{2}$ Graduate School of Pharmaceutical Sciences, The University of Tokyo, 7-3-1 Hongo, Bunkyo-ku, Tokyo 113-0033, Japan. Jian-Ming Lv and Dan Hu contributed equally to this work. Correspondence and requests for materials should be addressed to H.G. (email: tghao@jnu.edu.cn) or to I.A. (email: abei@mol.f.u-tokyo.ac.jp) or to X.-S.Y. (email: tyaoxs@jnu.edu.cn)
} 
T riterpenoids possess a variety of pharmacological activities and serve as an invaluable source of pharmaceuticals ${ }^{1}$. Ginsenosides and ganoderic acids are drug candidates ${ }^{2,3}$, and ginsenoside $\mathrm{Rg} 3$ has been clinically approved to treat cancers in China. However, extremely low amounts of these medicinal constituents in natural hosts have significantly hampered their applications ${ }^{4,5}$. Combinatorial biosynthesis is a feasible option to improve the yields of target compounds and expand chemical diversity, which however requires a thorough understanding of their biosynthesis. To date, only a few triterpenoids such as ginsenosides ${ }^{6}$ and betulinic acid ${ }^{7}$ have been demonstrated.

Fusidane-type antibiotics, a group of fungi-derived triterpenes, originate from protosta-17(20)Z,24-dien-3 $\beta$-ol (2) with unique tetracyclic skeleton, which is formed by enzymatic cyclization of (3S)-2,3-oxidosqualene ${ }^{8}$. Thus, after the formation of the tetracyclic C-20 protosteryl tertiary intermediate cation ${ }^{9}$, the cyclization reaction is terminated by elimination of $\mathrm{H}-17$ to form a double-bond between C-17 and C-20 without the backbone rearrangement. Helvolic acid $(\mathbf{1})^{10}$, fusidic acid ${ }^{11}$, and cephalosporin $\mathrm{P}_{1}{ }^{12}$ are the most representative fusidane-type antibiotics (Fig. 1a), all of which exhibit potent activity against Grampositive bacteria ${ }^{13}$. Amongst them, fusidic acid has been used to treat staphylococcal skin infections in Europe for nearly 60 years ${ }^{14}$. In the United States, the recent phase II clinical trial has demonstrated that fusidic acid showed comparable efficacy to linezolid for treatment of acute bacterial skin and skin structure infections (ABSSSIs) ${ }^{15}$. Along with the advancement of global antimicrobial resistance, fusidane-type antibiotics have drawn renewed attentions because they have no cross-resistance to commonly used antibiotics ${ }^{14,16}$.

The remarkable bioactivity and little cross-resistance of fusidane-type antibiotics have inspired many attempts to search for more active congeners. Great efforts have been focused on modifications of fusidane skeleton by means of chemical derivatization and biotransformation ${ }^{13,17}$. Exploring fusidane-type antibiotics biosynthesis could offer an opportunity to yield structurally novel derivatives, but the genetic and molecular basis for the biosynthesis of fusidane-type antibiotics remained obscure until 2009 when helvolic acid putative biosynthetic gene cluster consisting of nine genes (Fig. $1 \mathrm{~b}$ and Table 1) was identified from the genome sequence of Aspergillus fumigatus Af293 18,19 . Preliminary heterologous expression studies in Saccharomyces cerevisiae revealed that HelA (oxidosqualene cyclase, OSC), functioned to convert (3S)-2,3-oxidosqualene into protosta-17 (20)Z,24-dien-3 $\beta$-ol (2), which underwent dehydrogenation to form 3-keto (3) by HelC (short-chain dehydrogenase/reductase,
SDR), or oxidation to generate $4 \beta$-carboxylic acid (4) by HelB1 (cytochrome P450, P450) $)^{18}$. Nevertheless, it is still not clear whether the proposed nine-gene cluster is enough for helvolic acid biosynthesis, and if so, what the functions and reaction order of the six other genes are, and whether biosynthetic intermediates with more potent antibacterial activity than the end product helvolic acid exist.

In this work, we first introduce all the nine genes into the heterologous expression system A. oryzae NSAR $1^{20}$, and observe the production of helvolic acid $\left(\sim 20 \mathrm{mg} \mathrm{L}^{-1}\right)$, confirming that the putative gene cluster is sufficient for helvolic acid biosynthesis. Then, we determine the function of each gene and reaction sequences by reconstitution of the nine genes one by one in $A$. oryzae NSAR1, which enables us to isolate 21 derivatives and find three intermediates with more potent activity than helvolic acid. Remarkably, we observe an unusual C-4 demethylation process that is distinct from the sterol biosynthesis. These findings have provided a basis for constructing fusidane-type derivatives using biosynthetic approaches.

\section{Results}

Heterologous expression of the 9-gene cluster in A. oryzae. For convenience, we designated the reported helvolic acid gene cluster from A. fumigatus Af293 as the hel cluster and renamed all nine genes (Fig. $1 \mathrm{~b}$ and Table 1). In order to determine whether the proposed gene cluster is sufficient to produce helvolic acid, we constructed an A. oryzae NSAR1 transformant strain $\mathrm{AO} 21$ co-expressing all the nine genes. After AO21 cultured in the induction medium for 6 days, the mycelia extract was analyzed by high performance liquid chromatography (HPLC). As a result, an additional peak (1) $\left(\sim 20 \mathrm{mg} \mathrm{L}^{-1}\right)$ was detected (Fig. 2h). According to high resolution electrospray ionization mass spectroscopy (HRESIMS) and nuclear magnetic resonance (NMR), 1 was established as helvolic acid. Therefore, we demonstrated that the presumed hel cluster was sufficient for the biosynthesis of helvolic acid, setting the stage for uncovering the detailed biosynthetic assembly line for helvolic acid.

Stepwise reconstitution of helvolic acid biosynthesis. Previously, the functions of HelA (OSC), HelB1 (P450), and HelC (SDR) have been studied through reconstitution in S. cerevisiae $^{18}$. Consistently, we also observed the production of protosta-17(20) $Z$,24-dien-3 $\beta$-ol (2) and its 3-keto derivative 3 when coexpressing helA and helC in A. oryzae NSAR1 (Supplementary a

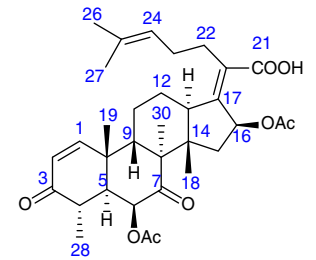

Helvolic acid (1)

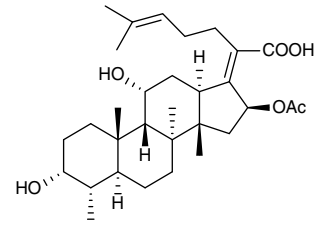

Fusidic acid

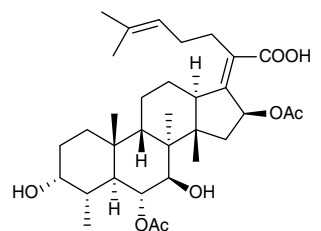

Cephalosporin $\mathrm{P}_{1}$

b

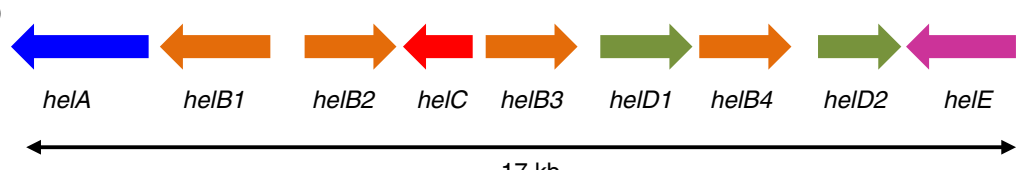

$17 \mathrm{~kb}$

Helvolic acid biosynthetic gene cluster from A. fumigatus Af293

Fig. 1 Representative fusidane-type antibiotics and biosynthetic gene cluster of helvolic acid (1). a Structures of helvolic acid (1), fusidic acid and cephalosporin $\mathrm{P}_{1}$; b Gene map of the helvolic acid gene cluster from A. fumigatus Af293 


\begin{tabular}{|lll|}
\hline Table 1 Putative functions of genes in the hel cluster \\
Gene & $\begin{array}{l}\text { Genbank accession } \\
\text { number }\end{array}$ & Putative function \\
\hline helA & XM_746263 & Oxidosqualene cyclase \\
helB1 & XM_746262 & Cytochrome P450 \\
helB2 & XM_746261 & Cytochrome P450 \\
helC & XM_746260 & Short-chain dehydrogenase/reductase \\
helB3 & XM_746259 & Cytochrome P450 \\
helD1 & XM_746258 & Acyltransferase \\
helB4 & XM_746257 & Cytochrome P450 \\
helD2 & XM_746256 & Acyltransferase \\
helE & XM_746255 & 3-Ketosteroid- $\Delta^{1}$-dehydrogenase \\
\hline
\end{tabular}

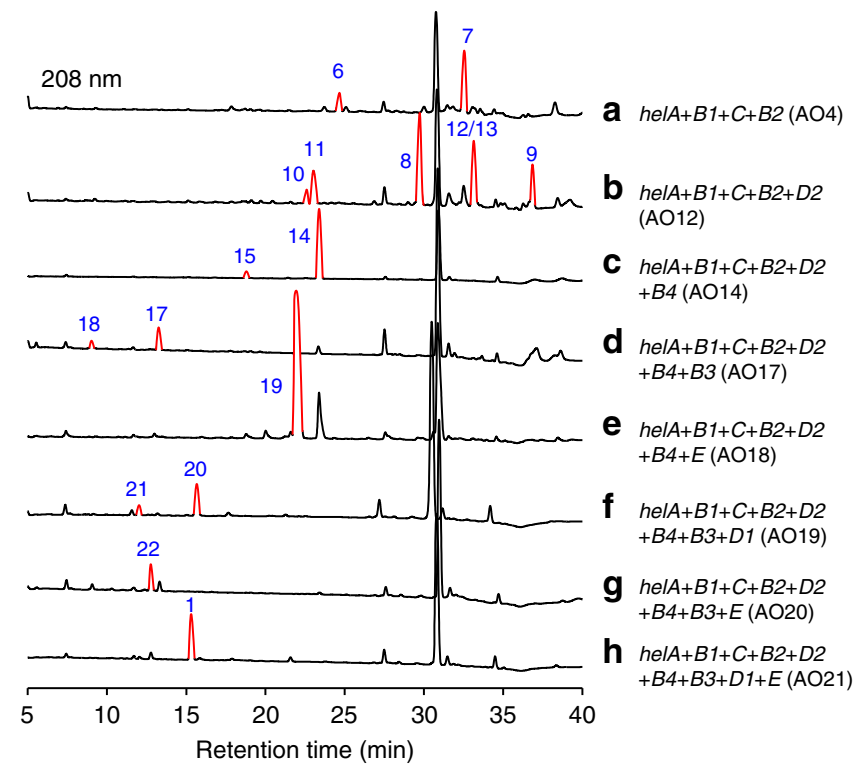

Fig. $2 \mathrm{HPLC}$ analysis of mycelia extract from different transformants. a $A$. oryzae harboring helA, helB1, helC and helB2; b $A$. oryzae harboring helA, helB1, helC, helB2 and helD2; c $A$. oryzae harboring helA, helB1, helC, helB2, helD2 and helB4; d A. oryzae harboring helA, helB1, helC, helB2, helD2, helB4 and helB3; e $A$. oryzae harboring helA, helB1, helC, helB2, helD2, helB4 and helE; $\mathbf{f} A$. oryzae harboring helA, helB1, helC, helB2, helD2, helB4, helB3 and helD1; $\mathbf{g} A$. oryzae harboring helA, helB1, helC, helB2, helD2, helB4, helB3 and helE; $\mathbf{h} A$. oryzae harboring helA, helB1, helC, helB2, helD2, helB4, helB3, helD1 and helE

Fig. 1c), and 4 upon co-expression of helA and helB1 (Supplementary Fig. 1b). Considering that HelB1 and HelC are responsible for the generation of $4 \beta$-carboxylic acid and 3-keto, respectively, it is conceivable that HelB1 and HelC could function together to give a $\beta$-keto carboxylic acid intermediate, which would undergo decarboxylation to remove the C- $4 \beta$ methyl group. In order to verify the hypothesis, we simultaneously introduced helA, helB1, and helC into A. oryzae NSAR1 to generate a transformant strain $\mathrm{AO}$. Upon gas chromatograph-mass spectrometer (GC-MS) analysis, an additional peak at $\mathrm{m} / z 410$ was observed in the mycelia extract of $\mathrm{AO} 3$, but not in the parent strain A. oryzae NSAR1 (Fig. 3 and Supplementary Fig. 2), which was also confirmed by HPLC analysis of AO3 (Supplementary Fig. 1a). HRESIMS and NMR analyses revealed that the additional peak was the demethylated product 5 (Fig. 4). These results clearly indicated that the HelB1 and HelC together could trigger the $4 \beta$-demethylation via oxidative decarboxylation (Fig. 5a),

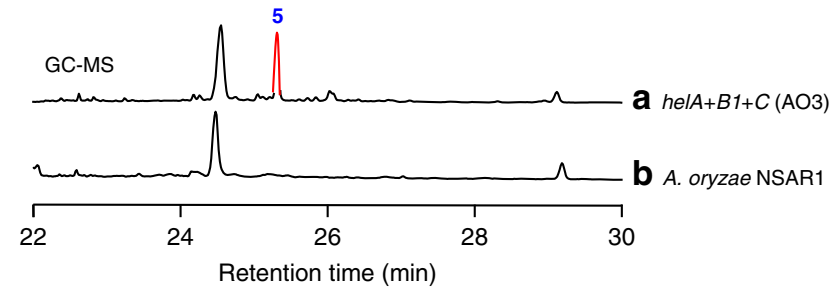

Fig. 3 GC-MS analysis of mycelia extract. a A. oryzae harboring helA, helB1 and helC; $\mathbf{b}$ A. oryzae

which is distinct from the sterol biosynthesis by $4 \alpha$ demethylation (Fig. 5b) ${ }^{21,22}$.

Assuming that $\mathbf{5}$ was the biosynthetic intermediate of helvolic acid, among the remaining six genes, only the P450 enzymes HelB2-4 and the dehydrogenase HelE might be engaged in the next modification. Thus we individually introduced helB2, helB3, helB4, and helE into the strain AO3 to generate transformants AO4-AO7. HPLC analyses of these strains showed that only introduction of helB2 (AO4) gave two additional peaks 6 and 7 (Fig. 2a and Supplementary Fig. 3). After isolation, both 6 and 7 were determined to bear $16 \beta$-hydroxyl group by NMR analysis, demonstrating that HelB2 (P450) is responsible for hydroxylation of the C-16 $\beta$ position (Fig. 4). 6 ought to be directly derived from 4 under the action of HelB2, while 7 could be generated by two plausible routes, i.e., hydroxylation of 5 by HelB2 or decarboxylation of $\mathbf{6}$ by HelC.

Similarly, supposing that $\mathbf{6}$ or 7 was the biosynthetic intermediate of helvolic acid, all the remaining five genes could be involved in the next reaction. We hence individually transformed each of the five genes into the strain $\mathrm{AO} 4$ to generate transformants $\mathrm{AO} 8-\mathrm{AO} 12$. We observed that only introduction of helD2 (AO12) led to the appearance of additional peaks in the HPLC profile (Fig. 2b and Supplementary Fig. 4). NMR structural characterization showed that $\mathbf{8}$ and $\mathbf{9}$ were the corresponding 16-O-acetylated products of 6 and 7, indicating that HelD2 (acyltransferase, AT) accounts for the acetylation of C-16 $\beta$ hydroxyl group (Fig. 4). For the generation of 9, two possible pathways may also exist as in the case of 7 . Along with $\mathbf{8}$ and 9 , four other products $10,11,12$, and 13 were also isolated in the strain AO12, which appeared as two pairs of epimers (Fig. 2b and Supplementary Fig. 4). These compounds were possibly derived from 8 and 9 by acetylation-elimination followed by the rearrangement of the double bond between C-17 and C-20 to C16 and $\mathrm{C}-17$, and quenching of the $\mathrm{C}-20$ cation with a water molecule (Fig. $5 c, d$ ). A similar mechanism has been employed in the biosynthesis of anditomin ${ }^{23}$ and pyrroindomycins ${ }^{24}$.

Owing to lack of hydroxyl group in the structures of $\mathbf{8}$ and $\mathbf{9}$, we next constructed the six gene-harboring transformants $\mathrm{AO} 13-\mathrm{AO} 15$ by means of individual introduction of helB3, helB4, and helE into the strain AO12. Only the strain AO14 containing helB4 afforded additional products 14 and 15 (Fig. 2c and Supplementary Fig. 5). 14 was featured with the carboxyl group at the C-20 position, indicating that HelB4 (P450) was responsible for converting C-20 methyl group to carboxylic acid (Fig. 4). And the minor product 15 was elucidated as the 3-keto reduced form of 14. Unexpectedly, we did not detect the HelB4oxidized product of $\mathbf{8}$ (compound $\mathbf{1 6}$ in Fig. 4) in AO14. To verify the possibility that the oxidized product $\mathbf{1 6}$ was quickly converted to 14 by $\mathrm{HelC}$, we established a helC-lacking transformant AO16 harboring helA, helB1, helB2, helD2, and helB4, and observed the accumulation of $\mathbf{1 6}$ in its mycelia extract (Supplementary Fig. 6). These results indicated that HelB4 could oxidize 8 into 16, which was then rapidly converted to $\mathbf{1 4}$ by HelC. 


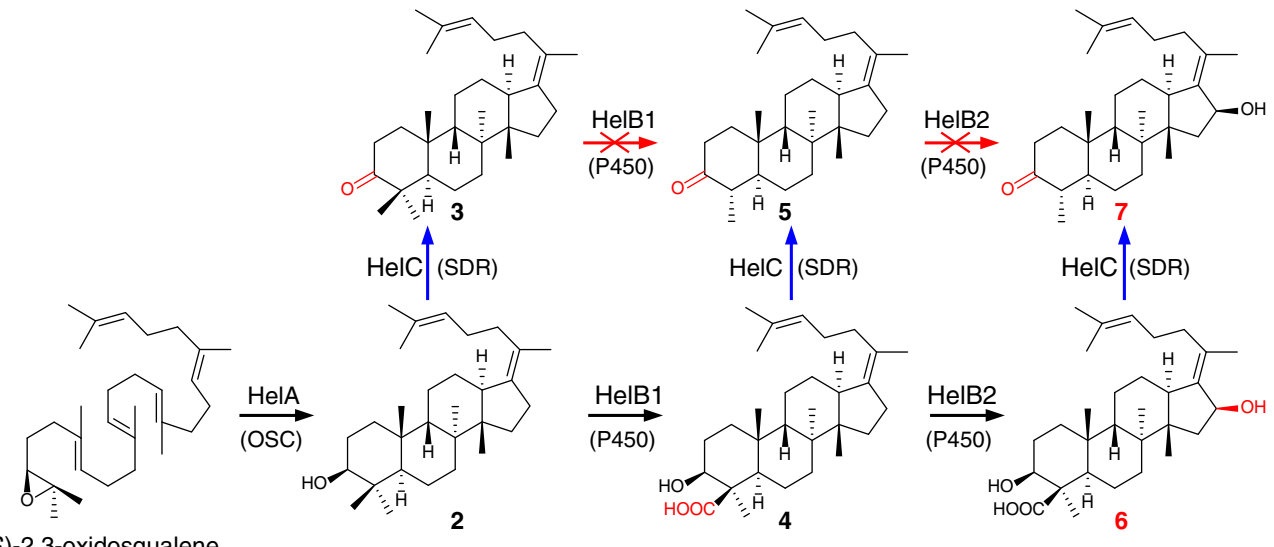

(3S)-2,3-oxidosqualene

2

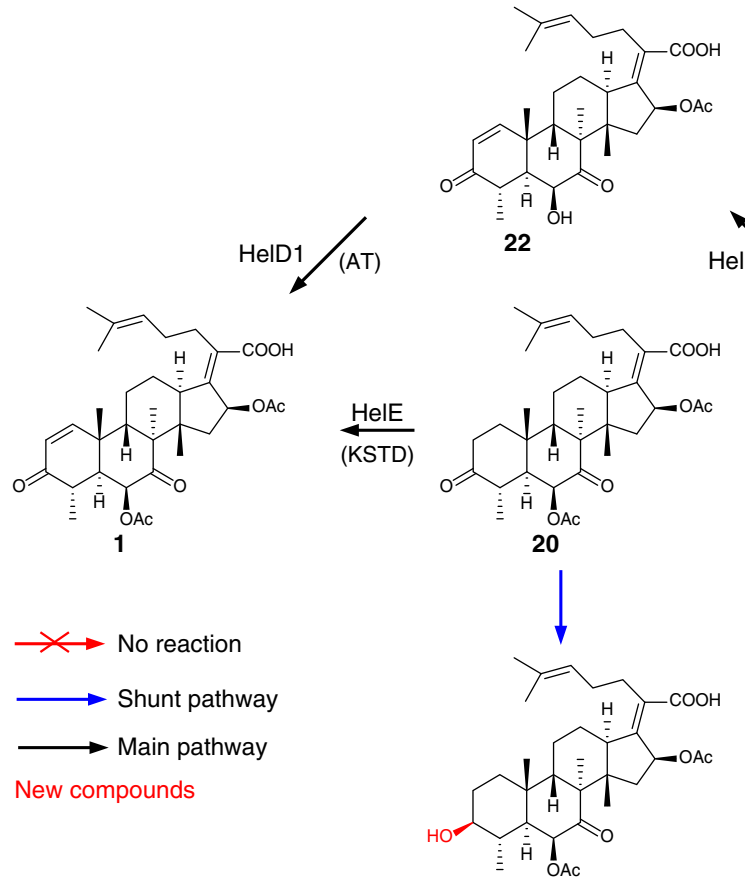

21
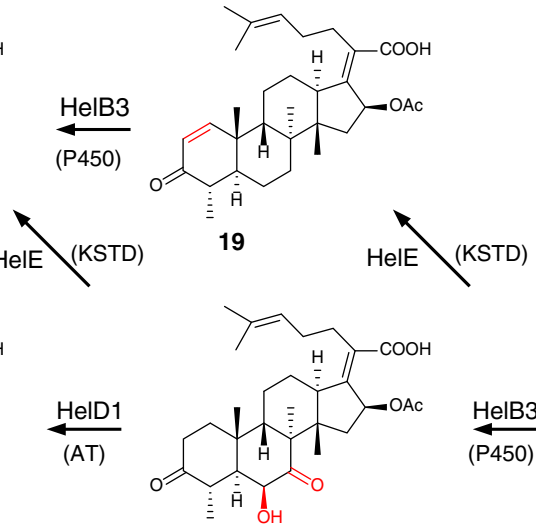

17

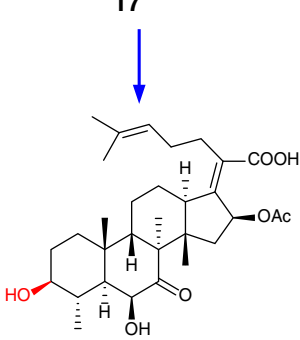

18

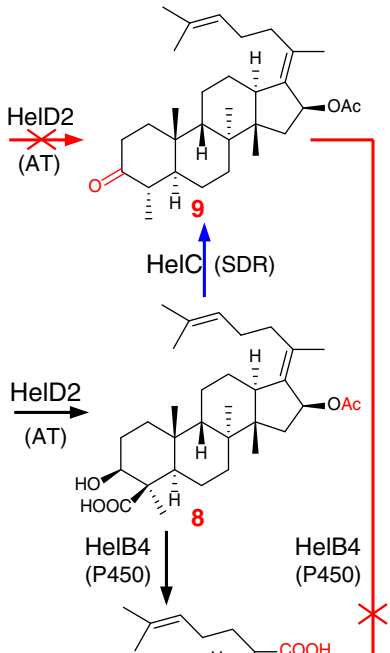

HelB4

(P450) 
a

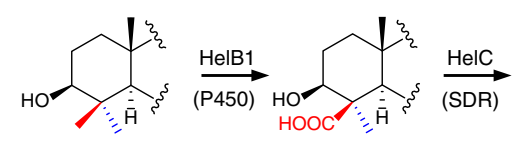<smiles>CCCCOC(=O)C1(C)C(=O)CCC(C)(C)C1CC</smiles>

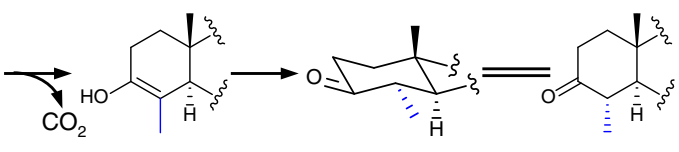

b

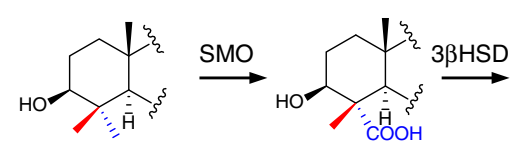

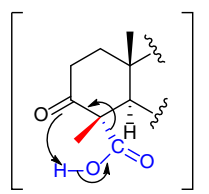

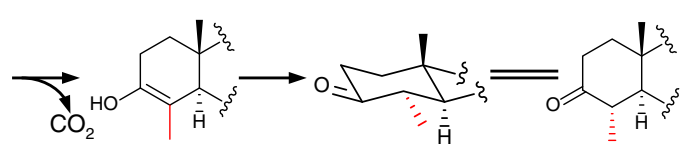

C

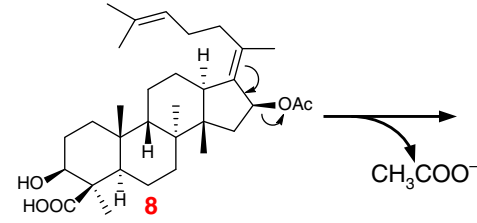

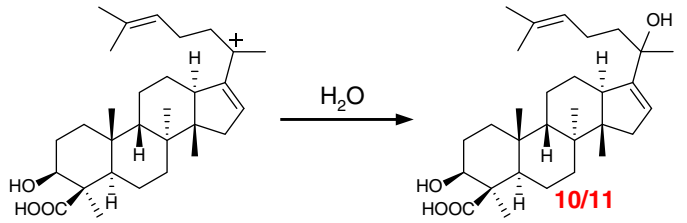

d
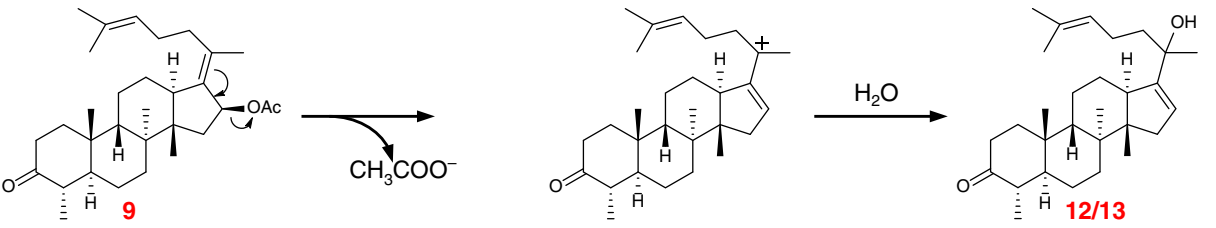

New compounds

Fig. 5 Proposed reaction mechanisms. a C-4 demethylation process in helvolic acid biosynthesis; b C-4 demethylation process in sterol biosynthesis; c Proposed mechanism for transformation of 8 to 10/11; $\mathbf{d}$ Proposed mechanism for transformation of $\mathbf{9}$ to $12 / \mathbf{1 3}$

( $\sim 20 \mathrm{mg} \mathrm{L}^{-1}$ ) (Fig. $2 \mathrm{~h}$ and Supplementary Fig. 9). Taken together, we have established the complete biosynthetic pathway for helvolic acid and obtained as many intermediates and shunt products as possible (Fig. 4).

An unusual C-4 demethylation process by HelB1 and HelC. The heterologous expression in A. oryzae NSAR1 revealed that HelB1 and HelC were responsible for the removal of the C- $4 \beta$ methyl group, which is distinct from the sterol C-4 demethylation process (Fig. 5a, b). To elucidate which occurs first: oxidation of C-4 $\beta$ methyl group by HelB1 or HelC-mediated dehydrogenation, $\mathrm{His}_{6}$-tagged HelC was expressed in Escherichia coli BL21(DE3) and purified (Supplementary Fig. 26). When we incubated HelC with substrate 4 in the presence of cofactor $\mathrm{NAD}^{+}$for $12 \mathrm{~h}$, the decarboxylated product 5 was detected, but not in the negative control containing inactive HelC (Fig. 6a, b). However, when we incubated HelB1-expressing strain $\mathrm{AO} 23$ with substrate 3, no product corresponding to $\mathbf{5}$ was detected based on liquid chromatograph-mass spectrometer (LC-MS) analysis (Supplementary Figs. 10 and 11). To rule out the possibility caused by the inactive expression of HelB1, we also fed this strain with substrate 2, and we did find its oxidized product 4 (Supplementary Figs. 12 and 13). These results unambiguously indicated that HelCmediated dehydrogenation follows HelB1-mediated oxidation of $\mathrm{C}-4 \beta$ methyl, which is similar to the reaction sequence in the sterol biosynthesis 21,22 .

In the sterol biosynthesis, the loss of two C-4 methyl groups is achieved by the successive action of C-4 sterol methyloxidase (SMO), C-3 sterol dehydrogenase (3ßHSD), and 3-keto reductase $(3 \mathrm{SR})^{21,22}$, while in the biosynthesis of helvolic acid, it is possible that the C-4 demethylation may take place at different stages as a result of the broad substrate range of HelC (Fig. 4). To verify these, we incubated recombinant HelC with substrates 6, 8, or 16, and we found that HelC could convert 6,8 and 16 to the

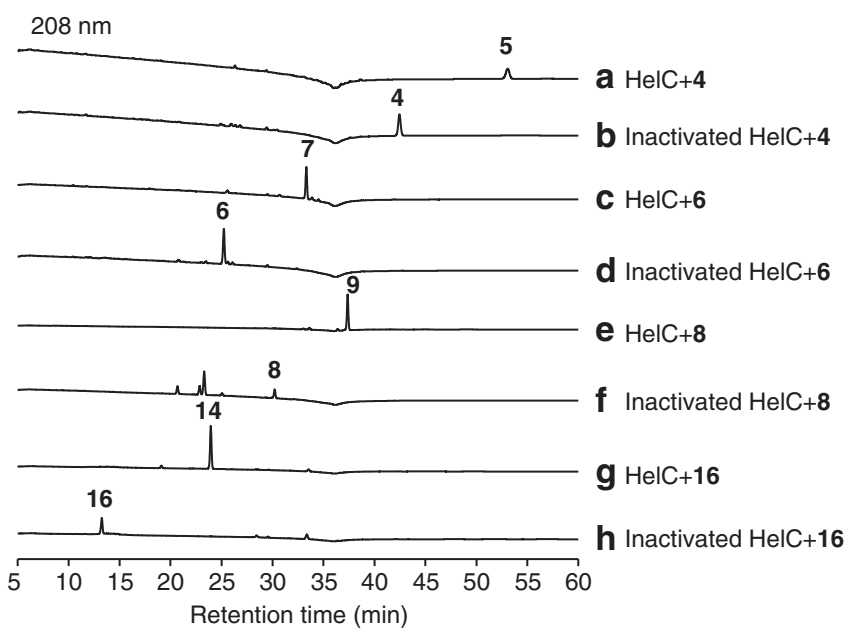

Fig. 6 HPLC analysis of in vitro enzymatic reactions. a 4 with $\mathrm{HelC}$; b 4 with inactivated HelC; c $\mathbf{6}$ with HelC; $\mathbf{d} \mathbf{6}$ with inactivated $\mathrm{HelC}$; $\mathbf{8}$ with HelC; $\mathbf{f}$ $\mathbf{8}$ with inactivated $\mathrm{HelC} ; \mathbf{g} \mathbf{1 6}$ with $\mathrm{HelC} ; \mathbf{h} \mathbf{1 6}$ with inactivated HelC

corresponding decarboxylated products 7, 9 and 14, respectively (Fig. 6c-h). These results demonstrated that HelC could work on multiple substrates to trigger the oxidative decarboxylation at different stages during the biosynthesis of helvolic acid.

The production of $\mathbf{7}, \mathbf{9}$, and $\mathbf{1 4}$ by HelC raised a doubt that the biosynthetic routes including HelB2-mediated conversion of 5 to 7, HelD2-mediated conversion of 7 to 9, and HelB4-mediated conversion of $\mathbf{9}$ to $\mathbf{1 4}$ may not exist. To test this hypothesis, feeding experiments for HelB2, HelB4 and HelD2 were carried out. The active expression of HelB2 in AO24, HelB4 in AO25, and HelD2 in AO26 were confirmed by feeding them with substrates 4, 8, and 6, respectively (Supplementary Figs. 14, 15, 
$18,19,22$, and 23). However, the transformant strains AO24 and AO25 could not generate $\mathbf{7}$ or $\mathbf{1 4}$ when supplemented with $\mathbf{5}$ and 9, respectively (Supplementary Figs. 16, 17, 20, and 21). These results suggested that the decarboxylation would prevent the substrates from modification by HelB2 and HelB4. Because 9 is hardly ionized, we could not confirm whether a very small amount of 7 was converted to 9 by AO26 using LC-MS analysis, but we did not observe any peak corresponding to 9 in the HPLC profile of AO26 fed with 7 (Supplementary Figs. 24 and 25), which indicates that HelD2 is likely to only accept the carboxylic derivative 6 (Fig. 4).

Three intermediates are more active than helvolic acid. In the course of our work, we obtained helvolic acid $\left(\sim 20 \mathrm{mg} \mathrm{L}^{-1}\right)$ and its 21 derivatives via reconstitution in $A$. oryzae, which provided an opportunity for structure-activity relationship analysis. We tested their inhibitory effects against Staphylococcus aureus 209P by the 2-fold dilution method (Table 2). Most compounds showed prominent inhibitory activities against this bacterium. Among them, 17, 19, and 22 showed even stronger activity than helvolic acid, and compound 22 exhibited the strongest antibacterial activity with minimum inhibition concentration (MIC) of $0.5 \mu \mathrm{g} \mathrm{mL}^{-1}$. The structure-activity relationship analysis revealed that both C-20 carboxyl group and 3-keto were important for the antibacterial activity, which is consistent with previous findings ${ }^{13}$. In addition, we firstly found that acetylation of the C-6 hydroxyl group was detrimental to the activity, as $\mathbf{1 7}$ and 22 exhibited higher activity than their corresponding acetylated products 20 and helvolic acid, respectively. These facts suggested that the end products are not always to be the most active molecules, and using biosynthetic approaches to access intermediates would be an effective way to obtain bioactive analogs.

\section{Discussion}

In this work, we have systematically unraveled the complete biosynthetic pathway for fusidane-type antibiotic helvolic acid by in vivo and in vitro experiments. Biosynthesis of helvolic acid includes nine steps from the universal triterpenoid progenitor (3S)-2,3-oxidosqualene (Fig. 4). Following the cyclization to the tetracyclic protosta-17(20)Z,24-dien-3 $\beta$-ol (2) by HelA, HelB1mediated and HelB2-mediated oxidation at C-4 and C-16, HelD2-dependent acetylation of 16-OH, oxidation of C-21 by HelB4, and HelC-dependent oxidative decarboxylation yield the fusidane skeleton 14, which is further modified in three additional steps mediated by HelB3, HelD1, and HelE to give helvolic acid (1) (Fig. 4). Compared with the late stages in the biosynthesis of helvolic acid, enzymes involved in the early stage modifications act in a relatively strict order (Fig. 4). Considering the fact that an intermediate featured with both C-20 carboxyl and C-16 $\beta$ hydroxyl could be readily converted to the off-pathway lactone

\begin{tabular}{|llll|}
\hline \multicolumn{4}{l}{ Table 2 Anti-Staphylococcus aureus activity of compounds } \\
Compound & MIC $\left(\boldsymbol{\mu} \mathbf{g ~ m L}^{-\mathbf{1}}\right)$ & Compound & MIC $\left(\boldsymbol{\mu g} \mathbf{~ m L}^{-\mathbf{1}}\right)$ \\
\hline Helvolic acid (1) & 2 & $\mathbf{1 2}$ & $>128$ \\
$\mathbf{2}$ & $>128$ & $\mathbf{1 3}$ & $>128$ \\
$\mathbf{3}$ & 128 & $\mathbf{1 4}$ & 2 \\
$\mathbf{4}$ & $>128$ & $\mathbf{1 5}$ & 8 \\
$\mathbf{5}$ & $>128$ & $\mathbf{1 6}$ & 64 \\
$\mathbf{6}$ & 32 & $\mathbf{1 7}$ & 1 \\
$\mathbf{7}$ & $>128$ & $\mathbf{1 8}$ & 32 \\
$\mathbf{8}$ & 64 & $\mathbf{1 9}$ & 1 \\
$\mathbf{9}$ & $>128$ & $\mathbf{2 0}$ & 16 \\
$\mathbf{1 0}$ & 16 & $\mathbf{2 1}$ & 32 \\
$\mathbf{1 1}$ & 32 & $\mathbf{2 2}$ & 0.5 \\
\hline
\end{tabular}

derivative ${ }^{13}$, it is reasonable that the hydroxylation of C-16 by HelB1 and subsequent acetylation by HelD2 should occur before the HelB3-mediated oxidation of C-21.

Different from the common sterol biosynthesis, biosynthesis of helvolic acid involves a distinct C-4 demethylation process (Fig. 5a, b). In the biosynthesis of sterol, it is the methyl group at C- $4 \alpha$ position that is oxidized to carboxylic acid by SMO and subsequently eliminated by oxidative decarboxylation under the action of $3 \beta \mathrm{HSD}$ to yield a transient enol intermediate (Fig. 5b), which should be rapidly converted to the 3-keto tautomer. In this process, the remaining methyl group originally at $4 \beta$ (axial) position will energetically favor epimerization to $4 \alpha$ (equatorial) position, which provides a second chance for oxidation by SMO and undergoes demethylation (Fig. 5b) ${ }^{25-27}$. Nevertheless, C-4 demethylation in fusidane-type antibiotics proceeds in an unusual manner though it is also achieved by oxidative decarboxylation. Feeding experiments using ${ }^{14} \mathrm{C}$ - and ${ }^{3} \mathrm{H}$-labeled mevalonic acid revealed that formation of fusidic acid involves the direct loss of the C- $4 \beta$ methyl group ${ }^{9}$. Reconstitution of the early stage biosynthesis of helvolic acid in $S$. cerevisiae revealed that HelB1 specifically converts C- $4 \beta$ methyl group into carboxyl group, and HelC catalyzes the formation of a 3-keto derivative ${ }^{18}$. In the present study, our experiments demonstrated that it is the methyl group at C- $4 \beta$ position that is oxidized by HelB1 and subsequently removed by a promiscuous SDR enzyme HelC (Fig. 5a). During C- $4 \beta$ demethylation, the remaining C- $4 \alpha$ methyl group originally at the equatorial position is energetically favorable and difficult to epimerize to the $\beta$ position for further oxidation by HelB1 (Fig. 5a). This could be the reason why fusidanetype antibiotics retain a single methyl group at their C-4 positions.

In addition, compared with the sterol biosynthesis, in which elimination of C- $4 \alpha$ methyl groups is achieved by the successive action of SMO and 3 $\beta \mathrm{HSD}$ (Fig. 5b), in the biosynthesis of helvolic acid, after HelB1-mediated oxidation of C- $4 \beta$ methyl, HelCcatalyzed decarboxylation can take place at different biosynthetic stages as a result of the promiscuity of HelC (Fig. 4). Interestingly, premature decarboxylation can prevent the substrates from being modified by HelB2, HelD2, and HelB4, implying that the C-4 $\beta$ carboxylic acid or the $3 \beta$-hydroxyl might be important for the recognition by these enzymes in the early stage. In contrast, HelE and HelB3 can only work on the decarboxylated products (Fig. 4). Considering that the reaction sites of these two enzymes are on the A-ring or near the A-ring, the axial C- $4 \beta$ carboxylic acid may sterically block the recognition by these enzymes.

There are four P450 enzymes in the biosynthetic gene cluster for helvolic acid. Among them, HelB3 is able to catalyze the dual oxidation of C- 6 and C-7, to which a similar mechanism is only observed in the fungal meroterpenoid terretonin biosynthesis ${ }^{28}$. Sequence alignment of the P450 enzymes from the helvolic acid gene cluster reported by us and those identified from Sarocladium oryzae $^{29}$ and Metarhizium anisopliae ${ }^{30}$ revealed some amino acid residues are specifically conserved in HelB3 homologs, but not in other P450 enzymes (Supplementary Fig. 27), which might account for its intriguing property. Additional X-ray crystal structure analysis is needed to provide deep insight into the enzymatic reaction mechanisms.

In this study, we obtained helvolic acid $\left(\sim 20 \mathrm{mg} \mathrm{L}^{-1}\right)$ and its 21 derivatives via reconstitution in $A$. oryzae NSAR1. We investigated the antibacterial activity of all these compounds and found that the C-20 carboxyl group and 3-keto are essential for their antimicrobial activity against $S$. aureus, which is consistent with the previous report ${ }^{13}$. It is worth noting that three intermediates 17, 19 and 22 exhibit more potent antibacterial activity than the end product helvolic acid. Based on the structural difference between 17 (MIC: $1 \mu \mathrm{g} \mathrm{mL}^{-1}$ ) and 20 (MIC: $16 \mu \mathrm{g} \mathrm{mL}^{-1}$ ), and 22 
(MIC: $0.5 \mu \mathrm{g} \mathrm{mL}^{-1}$ ) and helvolic acid (MIC: $2 \mu \mathrm{g} \mathrm{mL}^{-1}$ ), we could conclude that the free C-6 hydroxyl group is important for the antibacterial activity and further acetylation of $6 \beta-\mathrm{OH}$ is deleterious to the activity. These results also give a good example that in some cases, not all the biosynthetic genes in the cluster are designed to increase the biological activities of secondary metabolites, which is also shown in the cylindrocyclophane biosynthesis ${ }^{31}$. Our results suggested that the biosynthetic intermediates and shunt products might be an invaluable source of drug discovery. However, natural products obtained via the conventional method are usually the end products biosynthesized in the hosts ${ }^{32}$, and access to the biosynthetic intermediates is always difficult. Therefore, biosynthetic approaches, especially heterologous expression, could allow the isolation of as many intermediates as possible ${ }^{23}, 33$, which will be an effective way to obtain more active derivatives.

Increased emergence of bacterial resistance has evoked the attention to fusidane-type antibiotics ${ }^{34}$, and the unambiguous determination of function and reaction sequences of nine genes in the helvolic acid gene cluster will advance the generation of artificial fusidane analogs using synthetic biology. The distinct C4 demethylation mechanism identified in the helvolic acid biosynthesis could be used for generating single C-4 methylated triterpenoids. Moreover, the stepwise reconstitution of the helvolic acid biosynthesis in A. oryzae has enabled us to isolate twenty-two products and obtain three intermediates with more potent activity than helvolic acid. We believe that heterologous expression could not only be useful for exploration of biosynthetic pathway, but also a powerful approach to complement conventional isolation in terms of drug discovery.

In conclusion, we have systematically unraveled the complete biosynthetic pathway to helvolic acid, one of the most representative fusidane-type antibiotics with potent activity against Gram-positive bacteria, and elucidated an unusual C-4 demethylation process that is distinct from the common sterol biosynthesis. In addition, we have obtained, via reconstitution in $A$. oryzae NSAR1, helvolic acid and its 21 derivatives, three of which are more active than helvolic acid. These findings provide knowledge of the biosynthesis of fusidane-type antibiotics and demonstrate the applicability of synthetic biology to generate analogs of helvolic acid.

\section{Methods}

General materials and experimental procedures. Acetonitrile $\left(\mathrm{CH}_{3} \mathrm{CN}\right)$ was purchased from Oceanpak Alexative Chemical Co., Ltd. (Gothenburg, Sweden). Petroleum ether, ethyl acetate (EtOAc), and acetone were analytical-grade from Fine Chemical Co., Ltd. (Tianjin, China). Formic acid was purchased from Kemiou Chemical Reagent Co., Ltd. (Tianjin, China).

Primer synthesis and DNA sequencing were performed by Sangon Biotech Co., Ltd. (Shanghai, China). Plasmid purification kits and agarose gel DNA extraction kits were purchased from Sangon Biotech Co., Ltd. (Shanghai, China). PCR was carried out using a Mastercycler nexus gradient (Eppendorf, Hamburg, Germany) with KOD -Plus- polymerase (Toyobo, Osaka, Japan). In-Fusion ${ }^{\circledR}$ HD Cloning Kit and T4 DNA polymerase were purchased from Takara Bio Inc. (Dalian, China). Other DNA modification reagents were purchased from Thermo Fisher Scientific Inc. (Shenzhen, China).

GC-MS analysis was performed on Thermo Finnigan Trace GC Ultra equipped with a HP-5MS column $(0.25 \mathrm{~mm}$ i.d. $\times 30 \mathrm{~m}, 0.25 \mu \mathrm{m}$ film thickness $)$ and coupled with Thermo Finnigan Trace DSQ. The temperature of the ionization chamber was $300^{\circ} \mathrm{C}$ and the electron impact ionization voltage was $70 \mathrm{eV}$. The oven temperature began at $75^{\circ} \mathrm{C}$ and held for $5 \mathrm{~min}$, then increased to $230{ }^{\circ} \mathrm{C}$ at a rate of $20^{\circ} \mathrm{C} \mathrm{min}-1$ and stayed at $230^{\circ} \mathrm{C}$ for $2 \mathrm{~min}$. It then continued with a second ramp by rising at a rate of $10^{\circ} \mathrm{C} \mathrm{min}^{-1}$ to $310^{\circ} \mathrm{C}$ and held for $10 \mathrm{~min}$. Helium was used as the carrier gas.

HPLC and LC-MS were carried out on an Ultimate 3000 HPLC system (Dionex) and an amaZon SL ion trap mass spectrometer (Bruker) using electrospray ionization with a Cosmosil $5 \mathrm{C}_{18}$-MS-II column $(4.6 \mathrm{~mm}$ i.d. $\times 250$ $\mathrm{mm}, 5 \mu \mathrm{m}$; Nacalai Tesque, Inc., Japan). Elution was subjected to a linear gradient $\left[\mathrm{H}_{2} \mathrm{O}\right.$ containing $0.1 \%$ formic acid (A) and $\mathrm{CH}_{3} \mathrm{CN}$ containing $0.1 \%$ formic acid

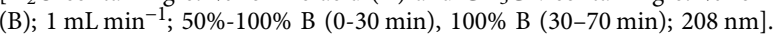

UV data, IR data and optical rotations were respectively measured on the JASCO V-550 UV/vis spectrometer, JASCO FT/IR-480 plus spectrometer, and JASCO P1020 digital polarimeter from JASCO International Co., Ltd., Tokyo, Japan. The HRESIMS data were obtained on a Micromass Q-TOF mass spectrometer (Waters Corporation, Milford, USA). 1D and 2D NMR spectra were obtained with Bruker AV 400 and Bruker AV 600 spectrometers (Bruker BioSpin Group, Faellanden, Switzerland) using the solvent signals $\left(\mathrm{CDCl}_{3}: \delta_{\mathrm{H}} 7.26 / \delta_{\mathrm{C}} 77.0\right.$; Pyridine- $\left.d_{5}: \delta_{\mathrm{H}} 7.21 / \delta_{\mathrm{C}} 123.5\right)$ as internal standards.

The semi-preparative HPLC was performed on an Ultimate 3000 HPLC system (Dionex) using a YMC-Pack ODS-A column $(10.0 \mathrm{~mm}$ i.d. $\times 250 \mathrm{~mm}, 5 \mu \mathrm{m}$; YMC Co., Ltd., Kyoto, Japan). Medium-pressure liquid chromatography (MPLC) was equipped with a dual-pump gradient system, a UV preparative detector, and a Dr. Flash II fraction collector system (Lisui E-Tech Co., Ltd., Shanghai, China). Column chromatography (CC) was performed with silica gel (200-300 mesh, Haiyang Chemical Co., Ltd., Qingdao, China) and ODS (50 $\mu \mathrm{m}$, YMC Co., Ltd., Tokyo, Japan)

Strains and media. Aspergillus oryzae NSAR1 (niaD $\left.{ }^{-}, s C^{-}, \Delta \operatorname{argB}, a d e A^{-}\right)^{20}$ served as the host for heterologous expression of genes. Transformants of $A$. oryzae NSAR1 were grown in $10 \mathrm{~mL}$ DPY medium (2\% dextrin, $1 \%$ polypeptone, $0.5 \%$ yeast extract, $0.05 \% \mathrm{MgSO}_{4} \cdot 7 \mathrm{H}_{2} \mathrm{O}, 0.5 \% \mathrm{KH}_{2} \mathrm{PO}_{4}$ ) for $1-2$ days at $28{ }^{\circ} \mathrm{C}$ and 150 $\mathrm{rpm}$. The cells were then transferred into Czapek-Dox (CD) medium $(0.3 \%$ $\mathrm{NaNO}_{3}, 0.2 \% \mathrm{KCl}, 0.05 \% \mathrm{MgSO}_{4} \cdot 7 \mathrm{H}_{2} \mathrm{O}, 0.1 \% \mathrm{KH}_{2} \mathrm{PO}_{4}, 0.002 \% \mathrm{FeSO}_{4} \cdot 7 \mathrm{H}_{2} \mathrm{O}, 1 \%$ polypeptone, $2 \%$ starch, $\mathrm{pH} 5.5$ ) to induce the expression of heterologous genes under the $a m y B$ promoter.

Standard gene engineering experiments were performed using E. coli $\mathrm{DH} 5 \alpha$ in LB medium supplemented with appropriate antibiotics, and E. coli BL21(DE3) was used for expression of HelC.

Construction of fungal expression plasmids. All the nine genes in the helvolic acid gene cluster were amplified from Aspergillus fumigatus Af293 genomic DNA with the primers listed in Supplementary Table 18. The helA gene was ligated into the SmaI-linearized pTAex3 vector using the In-Fusion ${ }^{\circledR}$ HD Cloning Kit to yield pTAex3-helA. The helB1 was cloned into the KpnI site of the pUSA vector to yield pUSA-helB1. The other seven genes were ligated into the pTAex3 vector digested with EcoRI or (and) KpnI to create pTAex3-helB2, pTAex3-helB3, pTAex3-helB4, pTAex3-helC, pTAex3-helD1, pTAex3-helD2, pTAex3-helE. pUSA-helC was constructed by ligation of the fragment amplified from pTAex3-helC using HelCEcoRI-F/HelC-EcoRI-R to the SmaI-digested pUSA vector.

pUSA-helB1-helC was constructed by ligation of the helC-containing fragment generated from the BamHI-digestion of pTAex3-helC to the BamHI-linearized pUSA-helB1. pAdeA-helB2, pAdeA-helB3, pAdeA-helB4, pAdeA-helE, pPTRIhelB3, and pPTRI-helE were constructed by the insertion of the fragments amplified from pTAex3-helB2, pTAex3-helB3, pTAex3-helB4, pTAex3-helE with the primers pTAex3-Pamy-F/pTAex3-Tamy-R into the XbaI-digested pAdeA or SmaI-digested pPTRI. For construction of plasmids harboring two or three genes, DNA fragments including the $a m y B$ promoter and terminator were amplified from pTAex3-based plasmids with the corresponding primers, and then ligated with the XbaI-digested pAdeA or HindIII-digested pPTRI using the In-Fusion ${ }^{\circledR} \mathrm{HD}$ Cloning Kit. All of the expression plasmids are listed in Supplementary Table 19.

Transformation of $\boldsymbol{A}$. oryzae NSAR1. The spore suspension of $A$. oryzae NSAR1 was inoculated into the $10 \mathrm{~mL}$ DPY medium and cultivated for two days at $28^{\circ} \mathrm{C}$ and $150 \mathrm{rpm}$. Then the cells were transferred into $100 \mathrm{~mL}$ DPY medium and continued growing for one day at $28^{\circ} \mathrm{C}$ and $150 \mathrm{rpm}$. Mycelia were collected by filtration, and cell walls were digested using $1 \%$ Yatalase (Takara) in $0.6 \mathrm{M}$ $\left(\mathrm{NH}_{4}\right)_{2} \mathrm{SO}_{4}, 50 \mathrm{mM}$ maleic acid, $\mathrm{pH} 5.5$ at $30^{\circ} \mathrm{C}$ for $3 \mathrm{~h}$. After removing residues by filtration, protoplasts were centrifuged at $1500 \mathrm{rpm}$ for $10 \mathrm{~min}$ and washed with Solution 2 (1.2 M sorbitol, $50 \mathrm{mM} \mathrm{CaCl} \cdot 2 \mathrm{H}_{2} \mathrm{O}, 35 \mathrm{mM} \mathrm{NaCl}, 10 \mathrm{mM}$ Tris- $\mathrm{HCl}$, $\mathrm{pH}$ 7.5), and then adjusted to $2 \times 10^{7}$ cells $\mathrm{mL}^{-1}$ in Solution 2. Mixtures of $200 \mu \mathrm{L}$ protoplasts solution and $<15 \mu \mathrm{L}$ plasmids $(10 \mu \mathrm{g})$ were incubated on ice for $30 \mathrm{~min}$, and subsequently $1.35 \mathrm{~mL}$ Solution 3 (60\% PEG4000, $50 \mathrm{mM} \mathrm{CaCl} \cdot 2 \mathrm{H}_{2} \mathrm{O}, 10 \mathrm{mM}$ Tris- $\mathrm{HCl}, \mathrm{pH} 7.5$ ) was added to the aliquot. After $20 \mathrm{~min}$ incubation at the room temperature, $10 \mathrm{~mL}$ Solution 2 was added, and then the mixture was subjected to centrifugation at $1500 \mathrm{rpm}$ for $10 \mathrm{~min}$. The precipitates were suspended in $200 \mu \mathrm{L}$ Solution 2 and spread on the lower selective medium $\left(0.2 \% \mathrm{NH}_{4} \mathrm{Cl}, 0.1 \%\right.$ $\left(\mathrm{NH}_{4}\right)_{2} \mathrm{SO}_{4}, 0.05 \% \mathrm{KCl}, 0.05 \% \mathrm{NaCl}, 0.1 \% \mathrm{KH}_{2} \mathrm{PO}_{4}, 0.05 \% \mathrm{MgSO}_{4} \cdot 7 \mathrm{H}_{2} \mathrm{O}, 0.002 \%$ $\mathrm{FeSO}_{4} \cdot 7 \mathrm{H}_{2} \mathrm{O}, 2 \%$ glucose and $1.2 \mathrm{M}$ sorbitol as well as $0.15 \%$ methionine, $0.1 \%$ arginine, $0.01 \%$ adenine, and $0.1 \mu \mathrm{g} \mathrm{mL}^{-1}$ pyrithiamine hydrobromide if necessary, $\mathrm{pH}$ 5.5) with $1.5 \%$ agar, and then covered with the selective upper medium containing $0.8 \%$ agar. The plates were incubated at $30^{\circ} \mathrm{C}$ for five to seven days. All of the transformants used in the work are listed in Supplementary Table 20.

Analysis and isolation of metabolites. To analyze the metabolites in different transformants, the seed broth cultured in $10 \mathrm{~mL}$ DPY medium for 2 days were inoculated into $100 \mathrm{~mL} \mathrm{CD}$ medium in a $500 \mathrm{~mL}$ flask at $28^{\circ} \mathrm{C}$ for $5-6$ days on a rotary shaker at $150 \mathrm{rpm}$. Then, mycelia were collected by filtration and extracted with acetone at the room temperature, and if necessary, the culture medium was extracted with EtOAc. The extract concentrated under reduced pressure was 
resuspended for analysis. For isolation of metabolites, extract was subjected to the column chromatography and further purification by semi-preparative HPLC (Supplementary Methods).

Heterologous expression and purification of HelC. The fragment coding HelC amplified from pTAex3-helC using SDR-NdeI-F/SDR-HindIII-R was cloned into the expression vector pET28a at the NdeI and HindIII sites in frame with an $N$ terminal $\mathrm{His}_{6}$-tag sequence. E. coli BL21(DE3) harboring pET28a-helC was inoculated into $10 \mathrm{~mL} \mathrm{LB}$ medium with $50 \mathrm{mg} \mathrm{L}^{-1}$ kanamycin and cultured at $37^{\circ} \mathrm{C}$ and $200 \mathrm{rpm}$ overnight, and then $1.5 \mathrm{~mL}$ seed broth was transferred into the 500 $\mathrm{mL}$ flask with $150 \mathrm{~mL} \mathrm{LB}$ medium and $50 \mathrm{mg} \mathrm{L}^{-1}$ kanamycin, and grew at $37^{\circ} \mathrm{C}$ and $200 \mathrm{rpm}$ until $\mathrm{OD}_{600}$ reached up to $0.5-0.7$. The gene expression was induced with $0.2 \mathrm{mM}$ isopropyl $\beta$-D-1-thiogalactopyranoside (IPTG) at $18^{\circ} \mathrm{C}$ for $12-16 \mathrm{~h}$. Cells were harvested by centrifugation at $4^{\circ} \mathrm{C}$ and $5000 \times g$ for $10 \mathrm{~min}$. The pellet was washed with $50 \mathrm{mM}$ Tris- $\mathrm{HCl}(\mathrm{pH} 8.0)$ and resuspended in lysis buffer (50 $\mathrm{mM}$ Tris- $\mathrm{HCl}, 200 \mathrm{mM} \mathrm{NaCl}, 5 \mathrm{mM}$ imidazole, $5 \%$ glycerol, $1 \%$ proteinase inhibitor cocktail, $0.1 \%$ lysozyme, $\mathrm{pH} 8.0$ ), followed by sonication on ice for $10 \mathrm{~min}$. After centrifugation at $4{ }^{\circ} \mathrm{C}$ and $10,000 \times g$ for $10 \mathrm{~min}$, the supernatant was further filtered through a $0.45 \mu \mathrm{m}$ filter to remove cell debris and other particulate materials. The filtrate was subjected to a Ni Sepharose ${ }^{\mathrm{TM}} 6$ Fast Flow (GE Healthcare Bio-Sciences AB, Uppsala, Sweden) affinity chromatography column equilibrated with binding buffer $(50 \mathrm{mM}$ Tris- $\mathrm{HCl}, 200 \mathrm{mM} \mathrm{NaCl}, 5 \mathrm{mM}$ imidazole, $5 \%$ glycerol, $\mathrm{pH} 8.0)$. After elution with wash solution $(50 \mathrm{mM}$ Tris- $\mathrm{HCl}, 200$ $\mathrm{mM} \mathrm{NaCl}, 20 \mathrm{mM}$ imidazole, $5 \%$ glycerol, $\mathrm{pH} 8.0$ ) to remove host proteins, the bound HelC was eluted with elution solution $(50 \mathrm{mM}$ Tris- $\mathrm{HCl}, 200 \mathrm{mM} \mathrm{NaCl}$, $300 \mathrm{mM}$ imidazole, $5 \%$ glycerol, $\mathrm{pH}$ 8.0). The fraction containing HelC was concentrated and buffer-exchanged using the Amicon centrifugal filter Ultracel ${ }^{\circledR}$-10K (Merck Millipore Ltd., Darmstadt, Germany). The purified HelC was flash frozen in liquid nitrogen and stored at $-80^{\circ} \mathrm{C}$ for further use. In the course of expression and purification, HelC was confirmed using SDS-PAGE. Protein concentration was quantified according to the Bradford method via Pierce ${ }^{\mathrm{TM}}$ BCA Protein Assay Kit (Thermo Fisher Scientific Inc., Rockford, USA) and using bovine serum albumin as the standard protein.

In vitro assays and feeding experiments. In vitro enzymatic reaction for $\mathrm{HelC}$ was carried out in a reaction mixture (final volume, $200 \mu \mathrm{L}$ ) containing $100 \mathrm{mM}$ Tris- $\mathrm{HCl}$ ( $\mathrm{pH} 8.0$ ), $5 \mathrm{mM}$ NAD trihydrate, 2.35-7.00 $\mu \mathrm{M}$ HelC, and $0.5 \mathrm{mM}$ substrate. Following incubation at $30^{\circ} \mathrm{C}$ overnight, the mixture was extracted with 200 $\mu \mathrm{L}$ EtOAc. After evaporation of solvents, the residue was resuspended in methanol for HPLC analysis.

The A. oryzae NSAR1 transformant strain only containing helB1, helB2, helB4, or helD2 was cultured in $10 \mathrm{~mL}$ DPY medium for 1-2 days, and then was inoculated into $50 \mathrm{~mL} \mathrm{CD}$ medium for expression of exogenous genes. After cultivation for 1 day, the culture was supplemented with $1.0 \mathrm{mg}$ of substrate dissolved in $20 \mu \mathrm{L}$ DMSO, and was further incubated for 5 days. Mycelia were harvested and extracted with acetone. After removal of solvents under reduced pressure, samples were dissolved in methanol for LC-MS analysis.

Antibacterial assay. In vitro antibacterial activity against Gram-positive strain Staphylococcus aureus $209 \mathrm{P}$ was evaluated using the 2 -fold dilution assay ${ }^{35}, 36$. The bacterial strain was inoculated on nutrient agar $(0.3 \%$ beef extract, $0.2 \%$ yeast extract, $1 \%$ peptone, $0.5 \% \mathrm{NaCl}$ and $1.5 \%$ agar, $\mathrm{pH} 7.5$ ) at $37^{\circ} \mathrm{C}$ for $1-2$ days, then cells were collected with normal saline and adjusted to a concentration of $10^{7}-10^{9}$ $\mathrm{mL}^{-1}$ as seed broth. Compounds were dissolved into DMSO and adjusted to $50 \mathrm{mg}$ $\mathrm{L}^{-1}$. A total of $100 \mu \mathrm{L}$ seed broth was added to $200 \mathrm{~mL}$ nutrient solution, and then $200 \mu \mathrm{L}$ of mixtures was transferred into the first test well of each line in the 96-well while $100 \mu \mathrm{L}$ was added in the other wells in the same line. $0.5 \mu \mathrm{L}$ sample solution was added into the first well with the concentration of $128 \mathrm{mg} \mathrm{L}^{-1}$, then $100 \mu \mathrm{L}$ was transferred to the second well until the twelfth well with the final concentration of $0.06 \mathrm{mg} \mathrm{L}^{-1}$. Tobramycin was used as the positive control and DMSO as the negative control. The 96 -well microtiter plates were placed at $37^{\circ} \mathrm{C}$ for $24 \mathrm{~h}$. The MIC was defined based on the minimal concentration in which no bacteria were observed.

Structural characterization. Compound 1: A white powder; HRESIMS (positive) $m / z 591.2932[\mathrm{M}+\mathrm{Na}]^{+}$(calcd for $\mathrm{C}_{33} \mathrm{H}_{44} \mathrm{O}_{8} \mathrm{Na}, 591.2934$ ), see Supplementary Fig. 28a; NMR spectra, see Supplementary Fig. 28b, c; The NMR data are in good agreement with those of $6 \beta, 16 \beta$-diacetyloxy-29-norprotosta-1,17(20)Z,24-trien3,7-dione-21-oic acid (helvolic acid ${ }^{37}$ ) (Supplementary Note 1).

Compound 2: A colorless oil; NMR spectra, see Supplementary Fig. 29 a, b; The NMR data are in good agreement with those of protosta-17(20)Z,24-dien-3 $\beta$-ol ${ }^{19}$ (Supplementary Note 2).

Compound 3: A colorless oil; HRESIMS (positive) $m / z 425.3793[\mathrm{M}+\mathrm{H}]^{+}$ (calcd for $\mathrm{C}_{30} \mathrm{H}_{49} \mathrm{O}, 425.3783$ ), see Supplementary Fig. 30a; NMR spectra, see Supplementary Fig. 30b, c; The NMR data are in good agreement with those of protosta-17(20)Z,24-dien-3-one ${ }^{18}$ (Supplementary Note 3).

Compound 4: A white powder; HRESIMS (positive) $\mathrm{m} / z 457.3665[\mathrm{M}+\mathrm{H}]^{+}$ (calcd for $\mathrm{C}_{30} \mathrm{H}_{49} \mathrm{O}_{3}, 457.3682$ ), see Supplementary Fig. 31a; NMR spectra, see Supplementary Fig. 31b-g; NMR data, see Supplementary Table 1; The NMR data are in agreement with those of $3 \beta$-hydroxy-protosta- $17(20) Z$,24-dien-29-oic acid [protosta-17(20)Z,24-dien-3 $\beta$-ol-29-oic acid ${ }^{18}$ ], but are slightly revised (Supplementary Note 4).

Compound 5: A colorless oil; HRESIMS (positive) $\mathrm{m} / z 411.3600[\mathrm{M}+\mathrm{H}]^{+}$ (calcd for $\mathrm{C}_{29} \mathrm{H}_{47} \mathrm{O}, 411.3627$ ), see Supplementary Fig. 32a; NMR spectra, see Supplementary Fig. 32b-g; NMR data, see Supplementary Table 2; The structure is as the same as that of 29-norprotosta-17(20)Z,24-dien-3-one [3-oxofusida-17(20) $Z$,24-diene ${ }^{38}$, the detailed NMR assignments of which are not available prior to the present study (Supplementary Note 5).

Compound 6: A white powder; $[\alpha]_{\mathrm{D}}^{23}+52.0\left(c 0.5, \mathrm{CHCl}_{3}\right)$; UV $\left(\mathrm{CH}_{3} \mathrm{OH}\right) \lambda_{\max }$ $(\log \varepsilon) 206$ (4.05), 252 (3.33); IR (KBr) $\nu_{\max } 3429,2947,2872,1697,1621,1455$, $1375,1027 \mathrm{~cm}^{-1}$; HRESIMS (positive) $\mathrm{m} / z 473.3622[\mathrm{M}+\mathrm{H}]^{+}$(calcd for $\mathrm{C}_{30} \mathrm{H}_{49} \mathrm{O}_{4}$, 473.3631), see supplementary Fig. 33a; NMR spectra, see Supplementary Fig. 33b-i; NMR data, see Supplementary Table 3; 6 is identified as $3 \beta, 16 \beta$-dihydroxyprotosta-17(20)E,24-dien-29-oic acid (Supplementary Note 6).

Compound 7: A colorless oil; $[\alpha]_{\mathrm{D}}^{23}+39.6\left(c 0.5, \mathrm{CHCl}_{3}\right)$; UV $\left(\mathrm{CH}_{3} \mathrm{OH}\right) \lambda_{\max }$ $(\log \varepsilon) 205$ (3.67), 248 (3.18); IR (KBr) $\nu_{\max } 3421,2941,2872,1705,1624,1463$, $1375,1046 \mathrm{~cm}^{-1}$; HRESIMS (positive) $\mathrm{m} / z 427.3572[\mathrm{M}+\mathrm{H}]^{+}\left(\right.$calcd for $\mathrm{C}_{29} \mathrm{H}_{47} \mathrm{O}_{2}$, 427.3576), see Supplementary Fig. 34a; NMR spectra, see Supplementary Fig. 34b-h; NMR data, see Supplementary Table 4; 7 is identified as $16 \beta$-hydroxy29-norprotosta-17(20)E,24-dien-3-one (Supplementary Note 7).

Compound 8: A yellowish powder; $[\alpha]_{\mathrm{D}}^{23}+53.4\left(c 0.5, \mathrm{CHCl}_{3}\right) ; \mathrm{UV}\left(\mathrm{CH}_{3} \mathrm{OH}\right)$ $\lambda_{\max }(\log \varepsilon) 206$ (3.85), $253(2.79) ; \mathrm{IR}(\mathrm{KBr}) \nu_{\max } 3445,2944,2875,1729,1705$, $1449,1378,1239,1027 \mathrm{~cm}^{-1}$; HRESIMS (positive) $\mathrm{m} / z 537.3557[\mathrm{M}+\mathrm{Na}]^{+}$(calcd for $\mathrm{C}_{32} \mathrm{H}_{50} \mathrm{O}_{5} \mathrm{Na}, 537.3556$ ), see Supplementary Fig. 35a; NMR spectra, see Supplementary Fig. 35b-h; NMR data, see Supplementary Table 5; 8 is identified as $16 \beta$-acetyloxy-3 $\beta$-hydroxy-protosta-17(20)E,24-dien-29-oic acid (Supplementary Note 8).

Compound 9: A colorless oil; $[\alpha]_{\mathrm{D}}^{23}+37.4\left(c\right.$ 0.5, $\left.\mathrm{CHCl}_{3}\right)$; UV $\left(\mathrm{CH}_{3} \mathrm{OH}\right) \lambda_{\max }$ $(\log \varepsilon) 207$ (3.65), 248 (3.05); IR (KBr) $\nu_{\max } 2950,2869,1708,1454,1373,1245 \mathrm{~cm}$ ${ }^{-1}$; HRESIMS (positive) $\mathrm{m} / z 491.3472[\mathrm{M}+\mathrm{Na}]^{+}$(calcd for $\mathrm{C}_{31} \mathrm{H}_{48} \mathrm{O}_{3} \mathrm{Na}$, 491.3501), see Supplementary Fig. 36a; NMR spectra, see Supplementary Fig. 36b-g; NMR data, see Supplementary Table 6; 9 is identified as $16 \beta$-acetyloxy29-norprotosta-17(20)E,24-dien-3-one (Supplementary Note 9).

Compound 10: A white powder; $[\alpha]_{\mathrm{D}}^{23}+136.0\left(c 0.5, \mathrm{CH}_{3} \mathrm{OH}\right) ; \mathrm{UV}\left(\mathrm{CH}_{3} \mathrm{OH}\right)$ $\lambda_{\max }(\log \varepsilon) 205$ (3.77), 246 (2.97); IR (KBr) $\nu_{\max } 3429,2965,2938,2851,1688$, $1466,1449,1375,1098,1027 \mathrm{~cm}^{-1}$; HRESIMS (positive) $\mathrm{m} / z 473.3615[\mathrm{M}+\mathrm{H}]^{+}$ (calcd for $\mathrm{C}_{30} \mathrm{H}_{49} \mathrm{O}_{4}, 473.3631$ ), see Supplementary Fig. 37a; NMR spectra, see Supplementary Fig. 37b-g; NMR data, see Supplementary Table 7; 10 is identified as $3 \beta, 20$-dihydroxy-protosta-16,24-dien-29-oic acid with the absolute configuration of C-20 unsolved (Supplementary Note 10).

Compound 11: A white powder; $[\alpha]_{\mathrm{D}}^{23}+109.2\left(c 0.5, \mathrm{CH}_{3} \mathrm{OH}\right) ; \mathrm{UV}\left(\mathrm{CH}_{3} \mathrm{OH}\right)$ $\lambda_{\max }(\log \varepsilon) 205$ (3.91), 249 (3.05); IR (KBr) $\nu_{\max } 3450,2965,2938,2872,1697$, $1469,1449,1375,1098,1027 \mathrm{~cm}^{-1}$; HRESIMS (positive) $\mathrm{m} / z 473.3611[\mathrm{M}+\mathrm{H}]^{+}$ (calcd for $\mathrm{C}_{30} \mathrm{H}_{49} \mathrm{O}_{4}, 473.3631$ ), see Supplementary Fig. 38a; NMR spectra, see Supplementary Fig. 38b-g; NMR data, see Supplementary Table 8; 11 is identified as 3 $\beta, 20$-dihydroxy-protosta-16,24-dien-29-oic acid with the absolute configuration of C-20 unsolved (Supplementary Note 11).

Compound 12: A yellowish powder; $[\alpha]_{\mathrm{D}}^{23}+80.0\left(c 0.5, \mathrm{CHCl}_{3}\right) ; \mathrm{UV}\left(\mathrm{CH}_{3} \mathrm{OH}\right)$ $\lambda_{\max }(\log \varepsilon) 205$ (3.84), 246 (2.78); IR (KBr) $\nu_{\max } 3459,2962,2941,2869,1702$, $1452,1375,1106 \mathrm{~cm}^{-1}$; HRESIMS (positive) $\mathrm{m} / z 427.3569[\mathrm{M}+\mathrm{H}]^{+}$(calcd for $\mathrm{C}_{29} \mathrm{H}_{47} \mathrm{O}_{2}, 427.3576$ ), see Supplementary Fig. 39a; NMR spectra, see Supplementary Fig. 39b-g; NMR data, see Supplementary Table 9; 12 is identified as 20-hydroxy-29-norprotosta-16,24-dien-3-one with the absolute configuration of C-20 unsolved (Supplementary Note 12).

Compound 13: A yellowish powder; $[\alpha]_{\mathrm{D}}^{23}+59.0\left(c\right.$ 0.5, $\left.\mathrm{CHCl}_{3}\right) ; \mathrm{UV}\left(\mathrm{CH}_{3} \mathrm{OH}\right)$ $\lambda_{\max }(\log \varepsilon) 206$ (3.87), 245 (2.48); IR (KBr) $\nu_{\max } 3442,2960,2936,2872,1702$, $1455,1381,1109 \mathrm{~cm}^{-1}$; HRESIMS (positive) $\mathrm{m} / z 427.3568[\mathrm{M}+\mathrm{H}]^{+}$(calcd for $\mathrm{C}_{29} \mathrm{H}_{47} \mathrm{O}_{2}, 427.3576$ ), see Supplementary Fig. 40a; NMR spectra, see Supplementary Fig. 40b-g; NMR data, see Supplementary Table 10; 13 is identified as 20-hydroxy-29-norprotosta-16,24-dien-3-one with the absolute configuration of C-20 unsolved (Supplementary Note 13).

Compound 14: A yellowish powder; $[\alpha]_{\mathrm{D}}^{23}+43.0\left(c 0.5, \mathrm{CH}_{3} \mathrm{OH}\right) ; \mathrm{UV}\left(\mathrm{CH}_{3} \mathrm{OH}\right)$ $\lambda_{\max }(\log \varepsilon) 206$ (3.54), 225 (3.45); IR (KBr) $\nu_{\max } 2957,2944,2872,1729,1708$, $1455,1373,1253 \mathrm{~cm}^{-1}$; HRESIMS (positive) $\mathrm{m} / z 521.3244[\mathrm{M}+\mathrm{Na}]^{+}$(calcd for $\mathrm{C}_{31} \mathrm{H}_{46} \mathrm{O}_{5} \mathrm{Na}$, 521.3243), see Supplementary Fig. 41a; NMR spectra, see Supplementary Fig. 41b-h; NMR data, see Supplementary Table 11; 14 is identified as 16 $\beta$-acetyloxy-29-norprotosta-17(20)Z,24-dien-3-one-21-oic acid

(Supplementary Note 14).

Compound 15: A yellowish powder; $[\alpha]_{\mathrm{D}}^{23}+39.6\left(c 0.5, \mathrm{CH}_{3} \mathrm{OH}\right) ; \mathrm{UV}\left(\mathrm{CH}_{3} \mathrm{OH}\right)$ $\lambda_{\max }(\log \varepsilon) 206$ (3.56), 222 (3.51); IR (KBr) $\nu_{\max } 3439,2971,2944,2869,1729$, $1708,1449,1375,1250,1033 \mathrm{~cm}^{-1}$; HRESIMS (positive) $\mathrm{m} / z 523.3408[\mathrm{M}+\mathrm{Na}]^{+}$ (calcd for $\mathrm{C}_{31} \mathrm{H}_{48} \mathrm{O}_{5} \mathrm{Na}, 523.3399$ ), see Supplementary Fig. 42a; NMR spectra, see Supplementary Fig. 42b-g; NMR data, see Supplementary Table 12; 15 is identified as $16 \beta$-acetyloxy-3 $\beta$-hydroxy-29-norprotosta-17(20)Z,24-dien-21-oic acid (Supplementary Note 15).

Compound 16: A yellowish powder; $[\alpha]_{\mathrm{D}}^{23}+35.6\left(c 0.5, \mathrm{CH}_{3} \mathrm{OH}\right) ; \mathrm{UV}\left(\mathrm{CH}_{3} \mathrm{OH}\right)$ $\lambda_{\max }(\log \varepsilon) 206$ (3.82), 226 (3.74); IR (KBr) $\nu_{\max } 3456,2968,2953,2869,1729$, $1466,1375,1267,1024 \mathrm{~cm}^{-1}$; HRESIMS (positive) $\mathrm{m} / z 545.3474[\mathrm{M}+\mathrm{H}]^{+}$(calcd for $\mathrm{C}_{32} \mathrm{H}_{49} \mathrm{O}_{7}, 545.3478$ ), see Supplementary Fig. 43a; NMR spectra, see Supplementary Fig. 43b-g; NMR data, see Supplementary Table 13; 16 is identified 
as $16 \beta$-acetyloxy-3 $\beta$-hydroxy-protosta-17(20)Z,24-dien-21,29-dioic acid

(Supplementary Note 16).

Compound 17: A yellowish powder; HRESIMS (positive) $\mathrm{m} / z 551.2987$ [M + $\mathrm{Na}]^{+}$(calcd for $\mathrm{C}_{31} \mathrm{H}_{44} \mathrm{O}_{7} \mathrm{Na}$, 551.2985), see Supplementary Fig. 44a; NMR spectra, see Supplementary Fig. 44b-g; NMR data, see Supplementary Table 14; The structure is as the same as that of $16 \beta$-acetyloxy- $6 \beta$-hydroxy-29-norprotosta-17(20) Z,24-dien-3,7-dione-21-oic acid (CAS: 1379525-35-5), the detailed NMR assignments of which are not available prior to the present study (Supplementary Note 17).

Compound 18: A yellowish powder; $[\alpha]_{\mathrm{D}}^{23}-16.2\left(c 0.5, \mathrm{CH}_{3} \mathrm{OH}\right)$; $\mathrm{UV}\left(\mathrm{CH}_{3} \mathrm{OH}\right)$ $\lambda_{\max }(\log \varepsilon) 207$ (3.86), 225 (3.75); IR (KBr) $\nu_{\max } 3432,2953,2938,2869,1708$ $1621,1387,1355,1267,1027 \mathrm{~cm}^{-1}$; HRESIMS (positive) $\mathrm{m} / z$ 553.3139 $[\mathrm{M}+\mathrm{Na}]^{+}$ (calcd for $\mathrm{C}_{31} \mathrm{H}_{46} \mathrm{O}_{7} \mathrm{Na}$, 553.3141), see Supplementary Fig. 45a; NMR spectra, see Supplementary Fig. 45b-g; NMR data, see Supplementary Table 15; 18 is identified as $16 \beta$-acetyloxy-3 $\beta, 6 \beta$-dihydroxy-29-norprotosta-17(20)Z,24-dien-7-one-21-oic acid (Supplementary Note 18)

Compound 19: A white powder; HRESIMS (positive) $\mathrm{m} / z$ 497.3238 $[\mathrm{M}+\mathrm{H}]^{+}$ (calcd for $\mathrm{C}_{31} \mathrm{H}_{45} \mathrm{O}_{5}$, 497.3267), see Supplementary Fig. 46a; NMR spectra, see Supplementary Fig. 46b-g; NMR data, see Supplementary Table 16; The structure is as the same as that of $16 \beta$-acetyloxy-29-norprotosta-1,17(20)Z,24-trien-3-one21-oic acid [3-oxo-16 $\beta$-acetoxyfusida-1,17(20)(16,21-cis),24-trien-21-oic acid ${ }^{39}$ ], the detailed NMR assignments of which are not available prior to the present study (Supplementary Note 19).

Compound 20: A white powder; HRESIMS (positive) $\mathrm{m} / z$ 593.3081 $[\mathrm{M}+\mathrm{Na}]^{+}$ (calcd for $\mathrm{C}_{33} \mathrm{H}_{46} \mathrm{O}_{8} \mathrm{Na}$, 593.3090), see Supplementary Fig. 47a; NMR spectra, see Supplementary Fig. 47b, c; The NMR data are in good agreement with those of $6 \beta, 16 \beta$-diacetyloxy-29-norprotosta-17(20)Z,24-dien-3,7-dione-21-oic acid (1,2dihydrohelvolic acid ${ }^{40}$ ) (Supplementary Note 20 ).

Compound 21: A white powder; $[\alpha]_{\mathrm{D}}^{23}-30.8\left(c 0.5, \mathrm{CH}_{3} \mathrm{OH}\right) ; \mathrm{UV}\left(\mathrm{CH}_{3} \mathrm{OH}\right) \lambda_{\max }$ ( $\log \varepsilon) 206$ (3.71), 223 (3.60); IR (KBr) $\nu_{\max } 3450,2977,2944,2872,1747,1440$, $1375,1259,1027 \mathrm{~cm}^{-1}$; HRESIMS (positive) $\mathrm{m} / z 573.3428[\mathrm{M}+\mathrm{H}]^{+}$(calcd for $\mathrm{C}_{33} \mathrm{H}_{49} \mathrm{O}_{8}, 573.3427$ ), see Supplementary Fig. 48a; NMR spectra, Supplementary Fig. 48b-g; NMR data, see Supplementary Table 17; 21 is identified as $6 \beta, 16 \beta-$ diacetyloxy-3 $\beta$-hydroxy-29-norprotosta-17(20)Z,24-dien-7-one-21-oic acid (Supplementary Note 21).

Compound 22: A white powder; HRESIMS (positive) $\mathrm{m} / z$ 527.3009 $[\mathrm{M}+\mathrm{H}]^{+}$ (calcd for $\mathrm{C}_{31} \mathrm{H}_{43} \mathrm{O}_{7}, 527.3009$ ), see Supplementary Fig. 49a; NMR spectra, see Supplementary Fig. 49b, c; The NMR data are in good agreement with those of $16 \beta$-acetyloxy-6 $\beta$-hydroxy-29-norprotosta-1,17(20)Z,24-trien-3,7-dione-21-oic acid (helvolinic acid ${ }^{37}$ ) (Supplementary Note 22).

Data availability. All relevant data are available within the article and its Supplementary Information and from the corresponding authors on request.

Received: 20 August 2017 Accepted: 18 October 2017

Published online: 21 November 2017

\section{References}

1. Dzubak, P. et al. Pharmacological activities of natural triterpenoids and their therapeutic implications. Nat. Prod. Rep. 23, 394-411 (2006).

2. Wong, A. S. T., Che, C.-M. \& Leung, K.-W. Recent advances in ginseng as cancer therapeutics: a functional and mechanistic overview. Nat. Prod. Rep. 32, 256-272 (2015)

3. Bishop, K. S. et al. From 2000 years of Ganoderma lucidum to recent developments in nutraceuticals. Phytochemistry 114, 56-65 (2015).

4. Moses, T., Pollier, J., Thevelein, J. M. \& Goossens, A. Bioengineering of plant (tri)terpenoids: from metabolic engineering of plants to synthetic biology in vivo and in vitro. New. Phytol. 200, 27-43 (2013).

5. Pollier, J., Moses, T. \& Goossens, A. Combinatorial biosynthesis in plants: a (p) review on its potential and future exploitation. Nat. Prod. Rep. 28, 1897-1916 (2011).

6. Wang, P. et al. Production of bioactive ginsenosides $\mathrm{Rh} 2$ and $\mathrm{Rg} 3$ by metabolically engineered yeasts. Metab. Eng. 29, 97-105 (2015).

7. Li, J. \& Zhang, Y. Increase of betulinic acid production in Saccharomyces cerevisiae by balancing fatty acids and betulinic acid forming pathways. Appl. Microbiol. Biotechnol. 98, 3081-3089 (2014).

8. Abe, I., Rohmer, M. \& Prestwich, G. D. Enzymatic cyclization of squalene and oxidosqualene to sterols and triterpenes. Chem. Rev. 93, 2189-2206 (1993).

9. Mulheirn, L. J. \& Caspi, E. Mechanism of squalene cyclization. The biosynthesis of fusidic acid. J. Biol. Chem. 246, 2494-2501 (1971)

10. Chain, E., Florey, H. W., Jennings, M. A. \& Williams, T. I. Helvolic acid, an antibiotic produced by Aspergillus fumigatus, mut. helvola Yuill. Brit. J. Exp. Path. 24, 108-119 (1943).

11. Godtfredsen, W. O., Jahnsen, S., Lorck, H., Roholt, K. \& Tybring, L. Fusidic acid: a new antibiotic. Nature 193, 987 (1962).
12. Burton, H. S. \& Abraham, E. P. Isolation of antibiotics from a species of Cephalosporium. Cephalosporins $\mathrm{P}_{1}, \mathrm{P}_{2}, \mathrm{P}_{3}, \mathrm{P}_{4}$ and $\mathrm{P}_{5}$. Biochem. J. 50, 168-174 (1951).

13. von Daehne, W., Godtfredsen, W. O. \& Rasmussen, P. R. Structure-activity relationships in fusidic acid-type antibiotics. Adv. Appl. Microbiol. 25, 95-146 (1979).

14. Fernandes, P. Fusidic acid: a bacterial elongation factor inhibitor for the oral treatment of acute and chronic staphylococcal infections. Cold Spring Harb. Perspect. Med. 6, a025437 (2016).

15. Craft, J. C. et al. A randomized, double-blind phase 2 study comparing the efficacy and safety of an oral fusidic acid loading-dose regimen to oral linezolid for the treatment of acute bacterial skin and skin structure infections. Clin. Infect. Dis. 52, S520-S526 (2011)

16. Wilson, D. N. Ribosome-targeting antibiotics and mechanisms of bacterial resistance. Nat. Rev. Microbiol. 12, 35-48 (2014).

17. Duvold, T., Sørensen, M. D., Björkling, F., Henriksen, A. S. \& RastrupAndersen, N. Synthesis and conformational analysis of fusidic acid side chain derivatives in relation to antibacterial activity. J. Med. Chem. 44, 3125-3131 (2001).

18. Mitsuguchi, $\mathrm{H}$. et al. Biosynthesis of steroidal antibiotic fusidanes: functional analysis of oxidosqualene cyclase and subsequent tailoring enzymes from Aspergillus fumigatus. J. Am. Chem. Soc. 131, 6402-6411 (2009).

19. Lodeiro, S. et al. Protostadienol biosynthesis and metabolism in the pathogenic fungus Aspergillus fumigatus. Org. Lett. 11, 1241-1244 (2009).

20. Jin, F. J., Maruyama, J., Juvvadi, P. R., Arioka, M. \& Kitamoto, K. Development of a novel quadruple auxotrophic host transformation system by $\operatorname{argB}$ gene disruption using adeA gene and exploiting adenine auxotrophy in Aspergillus oryzae. Fems. Microbiol. Lett. 239, 79-85 (2004).

21. Rahier, A. Dissecting the sterol C-4 demethylation process in higher plants. From structures and genes to catalytic mechanism. Steroids 76, 340-352 (2011).

22. Nes, W. D. Biosynthesis of cholesterol and other sterols. Chem. Rev. 111, 6423-6451 (2011).

23. Matsuda, Y., Wakimoto, T., Mori, T., Awakawa, T. \& Abe, I. Complete biosynthetic pathway of anditomin: nature's sophisticated synthetic route to a complex fungal meroterpenoid. J. Am. Chem. Soc. 136, 15326-15336 (2014).

24. Zheng, Q. et al. A linear hydroxymethyl tetramate undergoes an acetylationelimination process for exocyclic methylene formation in the biosynthetic pathway of pyrroindomycins. Org. Biomol. Chem. 15, 88-91 (2017).

25. Gachotte, D. et al. Characterization of the Saccharomyces cerevisiae ERG27 gene encoding the 3-keto reductase involved in C-4 sterol demethylation. Proc. Natl Acad. Sci. USA 96, 12655-12600 (1999).

26. Gachotte, D., Barbuch, R., Gaylor, J., Nickel, E. \& Bard, M. Characterization of the Saccharomyces cerevisiae ERG26 gene encoding the C-3 sterol dehydrogenase (C-4 decarboxylase) involved in sterol biosynthesis. Proc. Natl Acad. Sci. USA 95, 13794-13799 (1998).

27. Bard, M. et al. Cloning and characterization of ERG25, the Saccharomyces cerevisiae gene encoding C-4 sterol methyl oxidase. Proc. Natl Acad. Sci. USA 93, 186-190 (1996)

28. Matsuda, Y., Iwabuchi, T., Wakimoto, T., Awakawa, T. \& Abe, I. Uncovering the unusual D-ring construction in terretonin biosynthesis by collaboration of a multifunctional cytochrome P450 and a unique isomerase. J. Am. Chem. Soc. 137, 3393-3401 (2015)

29. Hittalmani, S., Mahesh, H. B., Mahadevaiah, C. \& Prasannakumar, M. K. De novo genome assembly and annotation of rice sheath rot fungus Sarocladium oryzae reveals genes involved in helvolic acid and cerulenin biosynthesis pathways. BMC Genomics 17, 271-283 (2016).

30. Sbaraini, N. et al. Secondary metabolite gene clusters in the entomopathogen fungus Metarhizium anisopliae: genome identification and patterns of expression in a cuticle infection model. BMC Genomics 17, 736-754 (2016).

31. Nakamura, H., Hamer, H. A., Sirasani, G. \& Balskus, E. P. Cylindrocyclophane biosynthesis involves functionalization of an unactivated carbon center. J. Am. Chem. Soc. 134, 18518-18521 (2012).

32. Koehn, F. E. \& Carter, G. T. The evolving role of natural products in drug discovery. Nat. Rev. Drug Discov. 4, 206-220 (2005).

33. He, Y. \& Cox, R. J. The molecular steps of citrinin biosynthesis in fungi. Chem. Sci. 7, 2119-2127 (2016).

34. Bergen, P. J., Landersdorfer, C. B., Lee, H. J., Li, J. \& Nation, R. L. "Old" antibiotics for emerging multidrug-resistant bacteria. Curr. Opin. Infect. Dis. 25, 626-633 (2012)

35. Sarikahya, N. B. \& Kirmizigül, S. Antimicrobial triterpenoid glycosides from Cephalaria scoparia. J. Nat. Prod. 73, 825-830 (2010).

36. Mencherini, T., Picerno, P., Scesa, C. \& Aquino, R. Triterpene, antioxidant, and antimicrobial compounds from Melissa officinalis. J. Nat. Prod. 70, 1889-1894 (2007).

37. Fujimoto, H., Negishi, E., Yamaguchi, K., Nishi, N. \& Yamazaki, M. Isolation of new tremorgenic metabolites from an Ascomycete Corynascus setosus. Chem. Pharm. Bull. 44, 1843-1848 (1996). 
38. von Daehne, W. \& Godtfredsen, W. O. Tetracyclic triterpenes as cholesterollowering and anti-atherosclerosis agents. US Patent No. 6,177,418, (2001).

39. Okuda, S., Sato, Y., Hattori, T. \& Wakabayashi, M. Isolation and structural elucidation of 3-oxo-16 $\beta$-acetoxyfusida-1,17(20)(16,21-cis),24-trien-21-oic acid. Tetrahedron Lett. 47, 4847-4850 (1968).

40. Lee, S.-Y., Kinoshita, H., Ihara, F., Igarashi, Y. \& Nihira, T. Identification of novel derivative of helvolic acid from Metarhizium anisopliae grown in medium with insect component. J. Biosci. Bioeng. 105, 476-480 (2008).

\section{Acknowledgements}

This work was mainly supported by grants from the National Natural Science Foundation of China $(3171101305,31670036,81422054,81373306)$, the 111 Project of Ministry of Education of the People's Republic of China (B13038), the JST/NSFC Japanese-Chinese Collaborative Research Program, Guangdong Natural Science Funds for Distinguished Young Scholar (S2013050014287), Natural Science Foundation of Guangdong Province (2014A030313389), Guangdong Special Support Program (2016TX03R280), Guangdong Province Universities and Colleges Pearl River Scholar Funded Scheme (Hao Gao, 2014), and K. C. Wong Education Foundation (Hao Gao, 2016), and a Grant-in-Aid for Scientific Research from the Ministry of Education, Culture, Sports, Science and Technology, Japan (JSPS KAKENHI Grant Number JP15H01836 and JP16H06443).

\section{Author contributions}

D.H., H.G., I.A. and X.-S.Y. designed the research. J.-M.L. and D.H. performed the experiments. J.-M.L., D.H., H.G., T.K., T.A., G.-D.C., C.-X.W., I.A. and X.-S.Y. analyzed the data, and wrote the paper.

\section{Additional information}

Supplementary Information accompanies this paper at doi:10.1038/s41467-017-01813-9.

Competing interests: The authors declare no competing financial interests.

Reprints and permission information is available online at http://npg.nature.com/ reprintsandpermissions/

Publisher's note: Springer Nature remains neutral with regard to jurisdictional claims in published maps and institutional affiliations.

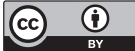

Open Access This article is licensed under a Creative Commons Attribution 4.0 International License, which permits use, sharing, adaptation, distribution and reproduction in any medium or format, as long as you give appropriate credit to the original author(s) and the source, provide a link to the Creative Commons license, and indicate if changes were made. The images or other third party material in this article are included in the article's Creative Commons license, unless indicated otherwise in a credit line to the material. If material is not included in the article's Creative Commons license and your intended use is not permitted by statutory regulation or exceeds the permitted use, you will need to obtain permission directly from the copyright holder. To view a copy of this license, visit http://creativecommons.org/ licenses/by/4.0/.

(C) The Author(s) 2017 